\title{
Capacity Configuration of Battery Energy Storage System for Photovoltaic Generation System Considering the High Charge- rate
}

\author{
Jiaming $\mathrm{Li}^{1}{ }^{1,}$, Ying $\mathrm{Qiao}^{1}$, Guojing $\mathrm{Liu}^{2}$, and Zongxiang $\mathrm{Lu}^{1}$ \\ ${ }^{1}$ State Key Lab of Control and Simulation of Power Systems and Generation Equipments, Dept. of Electrical Engineering, Tsinghua \\ University, Beijing 100084, China \\ ${ }^{2}$ Economic Institute of Technology, State Grid Jiangsu Electric Power Company, Nanjing 210005, China
}

\begin{abstract}
Battery energy storage system (BESS) is one of the important solutions to improve the accommodation of large-scale grid connected photovoltaic (PV) generation and increase its operation economy. However, the strong intra-day volatility and severe curtailment of PV power sets a high demand of BESS charge-rate that is a key factor in operation models but ignored in current planning researches. This paper proposes a BESS capacity configuration model for PV generation systems which takes BESS's ability to (dis)charge exceeds its rated power into account. The best charge-rate and power \& energy capacity of BESS are optimized by particle swarm optimization (PSO) algorithm. Through an analysis of the annual output statistics of PV power station in the northwest of China, the results show that when considering the high charge-rate of BESS, the optimal BESS capacity configuration rises and comprehensive income of the PV-BESS system will increase.
\end{abstract}

\section{Introduction}

With the energy transformation strategy proposed, photovoltaic (PV) generation installed capacity is increasing all over the word, and PV is becoming an important energy source in the future clean and renewable power system [1-3]. However, the development of $\mathrm{PV}$ is facing many challenges, the inherent features of PV such as strong volatility and randomness cause the difficulty to accommodate. The output characteristic of PV depends on the solar power, often mismatches with load demand, which gives rise to severe energy curtailment.

The BESS can help to absorb the electric energy in the PV peak period and release the electric energy in the low period, thus reduces the waste energy. With the continuous decrease of the cost of BESS, the integrated planning of energy storage and PV plants is seemed to be an effective, economical and reliable method, and some related research works have been done.

[4] gives the operation strategy and optimal capacity of BESS in a PV system based on the spectrum analysis of PV output. In [5], the method of interval estimation is used to predict probabilistic error of PV output, and the expected value of confidence level is obtained as the power allocation of BESS. [6] takes various scheduling modes into consideration, establishes the function of battery loss, then calculates the optimal BESS capacity.

However, in the existing researches on the capacity configuration of BESS for PV system, the charge-rate characteristics of BESS is often set to the rated value.
Thus, the potential capability of BESS to enhance accommodation is underestimated, which leads to the excessive investment of BESS. The charge-rate characteristic of BESS indicates that, many kinds of chemistry battery (such as graphite-LiFePO4, vanadium redox flow battery and so on) have the ability to charge or discharge at several times (often up to 5-10) of its rated power [7]. When BESS with good high-rate characteristics is charged or discharged at high rate, its energy conversion efficiency is almost the same [8], which shows the advantage of high-power conversion. The BESS with good high-rate characteristic is being developed and applied, so the high-rate (dis)charging ability of BESS needs to be considered in the configuration of BESS capacity for PV system.

In order to give the capacity configuration of BESS for PV system, an economic optimization model of PVBESS system is established, and the high-rate characteristics of BESS is considered in the model. The main works of the paper: Section 2 expounds the system structure and profit mode of PV generation system with BESS. In section 3, the high (dis)charge rate characteristics of BESS is given. The optimization model is given in Section 4. Section 5 shows a case study of a PV-BESS station in the northwest of China, and conducts a sensitivity analysis of various factors to BESS optimal (dis)charge rate. In Section 6 the conclusions are given.

\footnotetext{
* Corresponding author: jm-li19@mails.tsinghua.edu.cn
} 


\section{The technical and economic characteristics of PV-BESS generation system}

\subsection{The Structure of PV-BESS generation system}

Main structure of centralized PV-BESS generation system is shown in Fig. 1, and BESS system (including BESS and BESS converter) is in the dotted box. The PV generation can charge BESS and transmit electric energy to the grid through the PV converter. BESS can absorb the electric energy of PV when it is in the charging state through BESS converter, and transmits electric power to the grid when it works on the discharging state.

Since the charge/discharge state and input/output power of BESS can be controlled manually, its cooperation with the PV generation system can effectively slow down the fluctuation of equivalent PVBESS power generation, which improves the schedulability of PV system, and promotes the consumption.

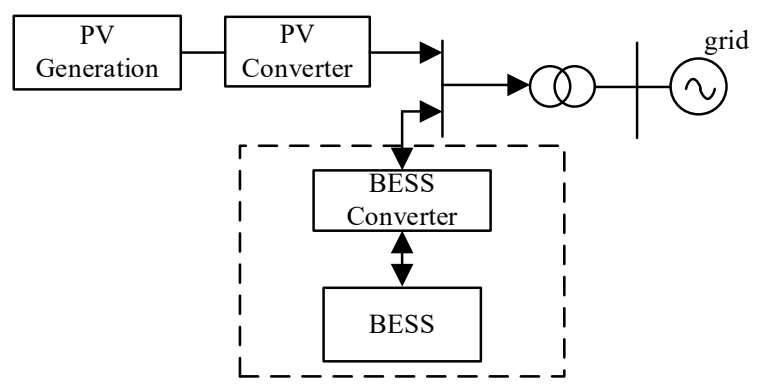

Fig. 1. Structure of PV-BESS system

\subsection{Profit model of PV-BESS generation system under the policy of restraint}

PV generation system gets profits from selling electricity to the grid. PV output power fluctuates strongly during the day, it has a strong power generation ability at noon on a sunny day. If all the electric power of PV is delivered to the grid at noon, normal grid dispatching will be affected seriously. Therefore, the power limitation measure is generally taken for PV system. The electric department formulates power limitation standards or PV plants, which means the power sold by PV system to the grid shall not exceed the power policy specified by the government department, the surplus power of PV will be wasted.

If additional BESS is set for PV system, BESS could absorb the surplus power of PV during the day time, then release electricity and sell it to the grid at night (Fig. 2). Thus, increase the income from electricity sales, however, the cost of BESS and BESS converter must be paid by the PV plants producer. In order to achieve better composite economic effects, it is necessary to allocate the capacity of BESS reasonably.

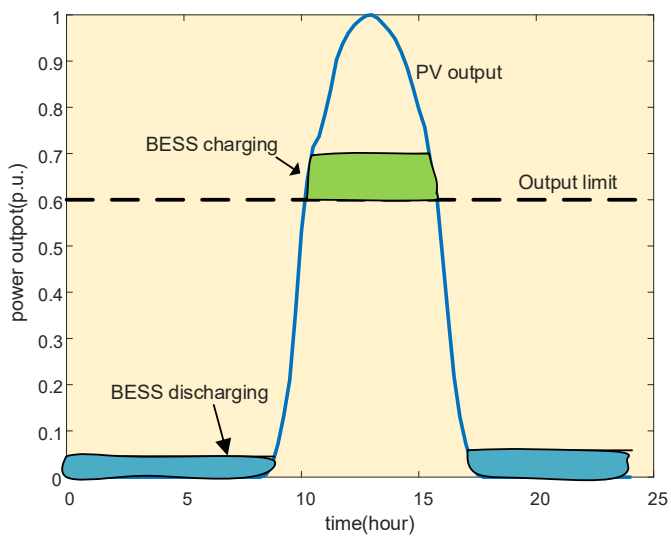

Fig. 2. Operation of PV-BESS system under the restraint policy

\section{High-rate characteristics of BESS}

Charge $\&$ discharge rate is the ratio of battery (dis)charge current to its rated capacity [9]. Generally, $\lambda$ is used to represent the ratio of battery charge and discharge current. For a $1200 \mathrm{mAh}$ battery, $\lambda=0.2$ represents (dis)charging at $240 \mathrm{~mA}(0.2$ times of $1200 \mathrm{mAh})$, and $\lambda=1.0$ represents $1200 \mathrm{~mA}$ ( 1 time of $1200 \mathrm{mAh}$ ). Under the condition of constant (dis)charge voltage, $\lambda$ can also represent the ratio of charge and discharge power to its rated power.

Supposing that the actual battery capacity won't change with its (dis)charging rate, the relationship between the continuous discharge time of a full-charged BESS and the discharging rate is shown in Fig. 3. (in the case of $\lambda=1$, the discharging time is $60 \mathrm{~min}$ ).

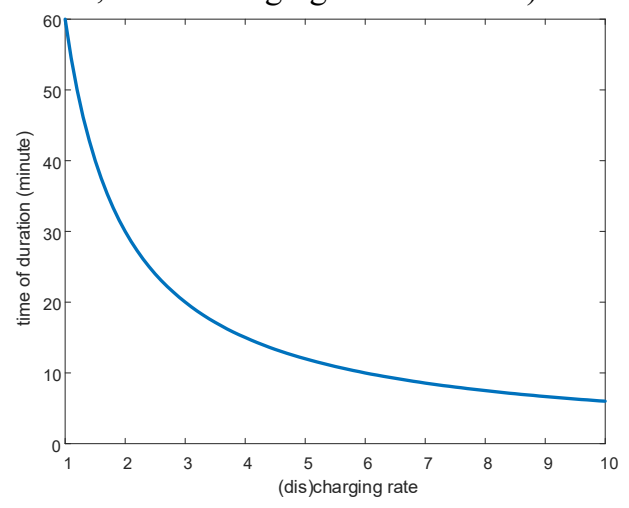

Fig. 3. The relationship between time duration and (dis)charging rate of BESS

Considering the energy conversion efficiency of BESS, the actual capacity that can be released by BESS is slightly smaller than in the ideal case, that is, the actual curve will be below the curve drew in .For most types of BESS, the larger the (dis)charge rate, the smaller capacity can be discharged or charged by BESS.

Using the data from the literature [10], the actual capacity of a particular lithium iron phosphate battery under different (dis)charge ratio is shown in Fig. 4, the capacity of energy that is released by the BESS under $\lambda=2$ shows $90 \%$ of the nominal capacity. 


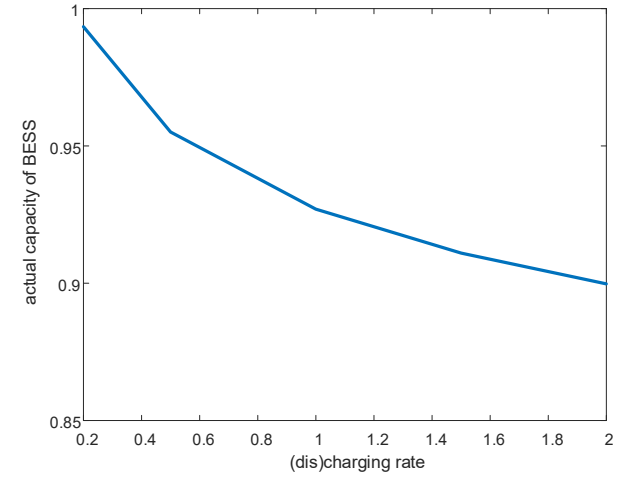

Fig. 4. The relationship between actual capacity and (dis)charging rate of BESS

\section{Optimization model}

The optimization model is built on the objection function of the extra annul income by setting BESS in a PV generation system. Because of the nonlinear nature of the optimization model, particle swarm optimization (PSO) algorithm is used to solve the problem.

\subsection{Optimization function}

\subsubsection{Optimization objective}

Taking the maximum extra annual income of PV generation system provided by the setting of BESS as the optimization target. The extra annual income includes the increased annual generation income and the construction $\&$ operation costs of BESS (equivalent to one year), and it can be expressed as followed:

$$
\max \left\{I_{n p}=\left(I_{\text {grid }}-I_{B E S S}\right)\right\}
$$

Where $I_{n p}$ is the extra annual income of PV generation provided by BESS (dollars/year), $I_{\text {grid }}$ is the extra annual power generation income of PV generation (dollars/year), $I_{B E S S}$ is the annual construction \& operation costs of BESS (dollars/year).

\subsubsection{Constraint condition}

The power \& capacity cost of BESS and the BESS converter cost matched with the highest (dis)charge rate $\left(\lambda_{\max }\right)$ are required to be paid for the construction of BESS system. $I_{B E S S}$ can be expressed as:

$$
I_{B E S S}=\left(C_{P} P_{B E S S}+C_{E} E_{B E S S}+\lambda_{\max } C_{\text {cov }} P_{B E S S}\right) / n_{B E S S}
$$

Where $C_{P}$ is sum construction and operation cost of unit BESS rated power (dollars/MW), $C_{E}$ is sum construction and operation cost of unit BESS rated energy capacity (dollars/MWh), $C_{\text {cov }}$ is sum construction and operation cost of unit BESS converter rated power (dollars/MW),
$P_{B E S S}$ is rated power of BESS (MW), $E_{B E S S}$ is rated energy capacity of BESS (MWh), $\lambda_{\max }$ is maximum charge/discharge rate of BESS, $n_{B E S S}$ is life span of BESS (year)

Increased revenue from electricity sales due to the construction of BESS can be expressed as:

$$
I_{\text {grid }}=C_{\text {grid }} E_{\text {grid }}
$$

Where $C_{\text {grid }}$ is electricity sale income of PV system (dollars/MWh), $E_{\text {grid }}$ is extra electricity sales capacity due to the establishment of BESS (MWh/year).

The BESS adopts the operation mode of charging once a day and discharging once a day to sell the electric energy stored in the daytime at night to the grid. Assuming that the electric power sold to the grid is limited to $P_{l}$, the electric output capacity of PV system on the $\mathrm{i}$ th day at time $t$ is $P_{\text {solar }}^{i}(t)$. If the rated power of BESS is set as $P_{B E S S}$, the maximum charge/discharge rate is set as $\lambda_{\max }$, supposing the configured energy storage capacity is large enough, the maximum energy that can be absorbed by BESS on the daytime is:

$$
E^{i}=\int_{P_{\text {solar }}^{i}(t)>P_{l}}\left(\min \left\{P_{\text {solar }}^{i}(t), P_{l}+\lambda_{\max } P_{B E S S}\right\}-P_{l}\right) d t
$$

Where $E^{i}$ is maximum energy can be absorbed on the $i$ th day (MWh), $P_{l}$ is power limit (maximum power sent to the grid) of PV-BESS system (MW), $P_{\text {solar }}^{i}(t)$ is output capacity of PV system on the $i$ th day at the time of $t$ (MW).

Considering the capacity limitation of BESS, as well as the charging \& discharging efficiency and the state of charge (SOC) limitation, the increased electricity sales capacity on this day due to the construction of BESS are as follows:

$$
E_{\text {grid }}^{i}=\eta_{c h} \eta_{d i s}\left(S O C_{\max }-S O C_{\min }\right) \min \left\{E^{i}, E_{B E S S}\right\}
$$

The annual extra electricity sales capacity can be expressed as:

$$
E_{\text {grid }}=\sum_{i=1 d}^{i=365 d} E_{\text {grid }}^{i}
$$

\subsection{Solving algorithm}

Because the objective function has nonlinear term, particle swarm optimization (PSO) algorithm is used. The core of PSO algorithm is to make use of the individual information sharing in the group, so that the movement of the whole group can generate a process from disorder to order in the solution space, so as to obtain the optimal solution of the problem. PSO algorithm is suitable for solving nonlinear mathematical programming problems 
without analytic expressions. Let the capacity of BESS $\left(E_{B E S S}\right)$ and the maximum (dis)charge rate set for BESS be the variables, and the solving flow chart is shown in Fig. 5.

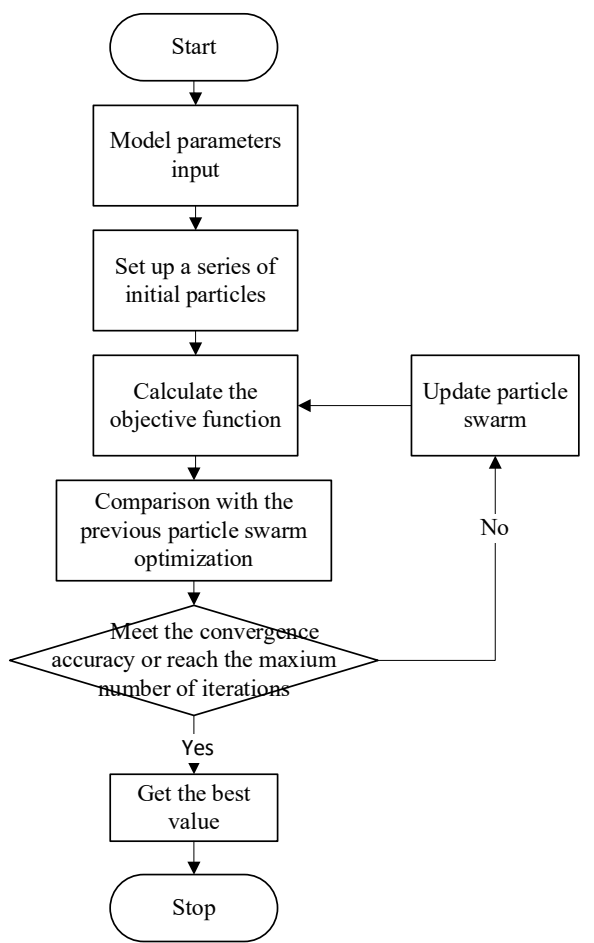

Fig. 5. Solving flow chart

\section{Case study}

\subsection{Parameters of the case}

Based on the annual actual power generation data (sampling interval is $15 \mathrm{~min}, 35040$ sample points totally) of a 100MW PV power station in Inner Mongolia, the above-mentioned maximum annual income model is used for simulation calculation, and the optimal capacity of energy storage device is compared under different maximum charge-rate of energy storage, and the sensitivity analysis of power station revenue is carried out. The BESS considered in this paper is lithium-ion battery, and the simulation parameters are set as Table 1:

Table 1. Value of parameters

\begin{tabular}{|c|c|c|c|}
\hline Parameter & Value & Parameter & Value \\
\hline$P_{l}$ & $50 \mathrm{MW}$ & $\begin{array}{c}S O C_{\max } \\
S O C_{\min }\end{array}$ & $\begin{array}{l}0.9 \\
0.1\end{array}$ \\
\hline$P_{B E S S}$ & $5 \mathrm{MW}$ & $\eta_{c h}, \eta_{d i s}$ & 0.9 \\
\hline$E_{B E S S}$ & $0-100 \mathrm{MWh}$ & $C_{P}$ & $1 * 10^{5} \$ / \mathrm{MW}$ \\
\hline$\lambda_{\max }$ & $1-5$ & $C_{E}$ & $\begin{array}{c}1.5^{*} 10^{5} \$ / \mathrm{M} \\
\mathrm{Wh}\end{array}$ \\
\hline
\end{tabular}

\begin{tabular}{|c|c|c|c|}
\hline$n_{\text {BESS }}$ & 15 years & $C_{\text {cov }}$ & $1 * 10^{5} \$ / \mathrm{MW}$ \\
\hline$C_{\text {grid }}$ & $90 \$ / \mathrm{MWh}$ & & \\
\hline
\end{tabular}

\subsection{Result analysis}

If taking both $\lambda_{\max }$ and $E_{B E S S}$ as the optimization variables, the result of the optimization is $\lambda_{\max }=2.6$, $E_{B E S S}=54.2 \mathrm{MWh}$, and the extra annual income is $I_{n p}=11.6\left(10^{4} \$\right)$. If $\lambda_{\max }$ is fixed in the optimization model, Fig. 6 shows the variation trend of optimal BESS capacity \& income with different $\lambda_{\max }$. The optimal capacity allocation of BESS increases with the increase of the maximum (dis)charge rate, and finally tends to be stable. Meanwhile, the benefits of BESS for PV system increase first and then decrease, and achieve the maximum at $\lambda_{\max }=2.6$. Compared with the case of the high-rate (dis)charge characteristics is ignored $\left(\lambda_{\max }=1\right.$, $E_{B E S S}=24.1 \mathrm{MWh}, I_{n p}=6.0^{*} 10^{4} \$$ ), the total annual income increases $93.3 \%$.

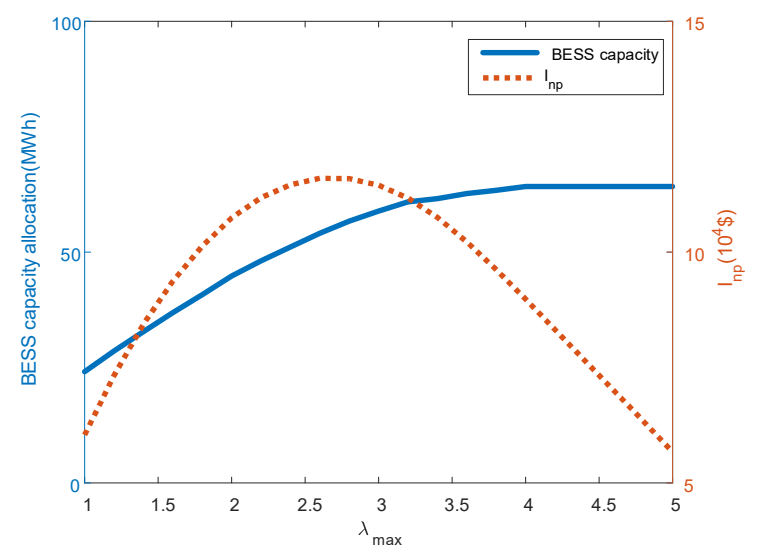

Fig. 6. Variation trend of optimal BESS capacity \& income with different $\lambda_{\max }$

\subsection{Sensitivity analysis}

The government's policy of limiting PV power sent to the grid affects the space in which BESS can play a role in increasing the generating capacity of PV stations, and the cost of BESS affects the economy of its installation and operation. Therefore, $P_{l}$ and $C_{E}$ are the two key factors that influence the optimization results. Fig. 7, Fig. 8 separately express the variation trend of optimal $\lambda_{\max }$ with different $P_{l}$ and $C_{E}$, when $P_{l}$ declines or $C_{E}$ is reduced, the optimal $\lambda_{\max }$ rises. PV stations with severe power limit needs higher charge rate, and with the cost reduction of BESS, battery with higher (dis)charge rate is more economical. 


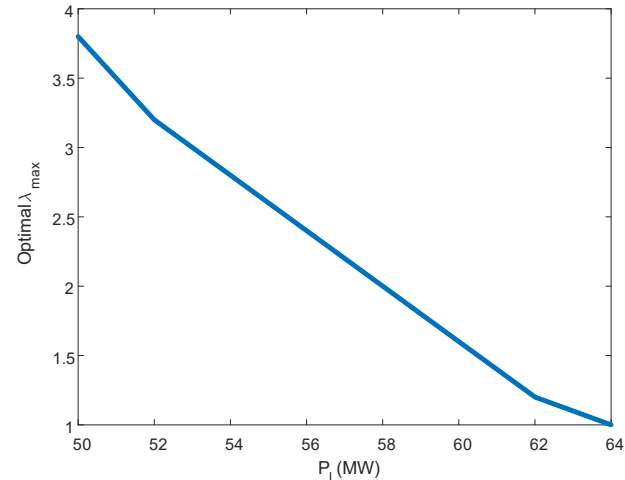

Fig. 7. Variation trend of optimal $\lambda_{\max }$ with different $P_{l}$

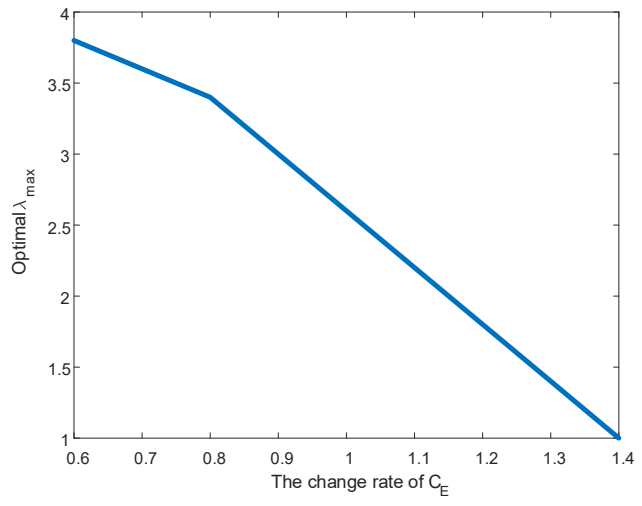

Fig. 8. Variation trend of optimal $\lambda_{\max }$ with different $C_{E}$

\section{Conclusion}

This study set out to calculate the most economical capacity of BESS for a PV generation considering the influence of (dis)charge-rate, where an optimization model is established. The results show that with considering the characteristics of high (dis)charge rate of BESS, both the BESS configuration capacity and comprehensive income of PV generation system will increase, especially in the PV station with severe curtailment. Besides, with trend of BESS cost decreasing, the optimal charge rate will rise, with which PV generation system will receive higher benefits.

This work is supported by the State Grid Technology Project "Research and Application of Large-scale Energy Storage Planning Technology for Received-end Power Grid". (5102201918309A-0-0-00)

\section{Reference}

1. ZHAO Bo, ZHANG Xuesong, HONG Bowen. Energy penetration of large-scale distributed photovoltaic sources integrated into smart distribution network[J]. Electric Power Automation Equipment, 2012,32(8): 95-100.

2. CHEN Shuyong, BAO Hai, WU Chunyang, et al . Direct grid-tie power control method for distributed photovoltaic generation $[\mathrm{J}]$. Proceedings of the CSEE, 2011, 30(10): 6-11.

3. TIAN Qi, ZHAO Zhengming, DENG Y, et al . Simulation and experimental study about reverse model of photovoltaic cells $[\mathrm{J}]$. Proceedings of the CSEE, 2011, 31(23): 121-128.

4. CHEN Gang, YUAN Yue, FU Zhixin. Application of storage battery to restrain the photovoltaic power fluctuation[J]. Proceedings of the CSU-EPSA, 2014, 26(2): 27-31.

5. LIN Shaobo, HAN Minxiao, ZHAO Guopeng, et al. Capacity allocation of energy storage in distributed photovoltaic power system based on stochastic prediction error $[\mathrm{J}]$. Proceedings of the CSEE, 2013,33(4): 25-33.

6. XU Yan, HE Chen, FU Chao, et al. Analysis of energy storage capacity allocation considering optimal economy of PV \& Battery cogeneration system under different scheduling modes[J]. Acta Energiae Solaris Sinica,2019,40(6): 1632-1640.

7. John Wang, Ping Liu, Jocelyn Hicks-Garner, et al. Cycle-life model for graphite-LiFePO4 cells[J]. Journal of Power Sources, 196(2011):3942-3948.

8. LI Shujuan, LI Xinran, HUANG Jiyuan, et al. Capacity Configuration of Lithium Titanate Battery in Whole Grid Frequency Regulation Based on Rate Characteristic $[\mathrm{J}]$. High Voltage Engineering,44(1):145-151.

9. Aghamohammadi M R, Abdolahinia H. A new approach for optimal sizing of battery energy storage system for primary frequency control of islanded Microgrid. International Journal of Electrical Power \& Energy Systems, 2014, 54(01):325-333.

10. TANG Jin XU Guofeng LI Jianling. Study on cycling performance of lithium iron phosphate battery at different discharge rates $[\mathrm{J}]$. Nonferrous Metals Science and Engineering,8(5):95-102. 